\title{
Accurate ionic forces and geometry optimization in linear-scaling density-functional theory with local orbitals
}

\author{
Nicholas D. M. Hine, ${ }^{1, *}$ Mark Robinson, ${ }^{2}$ Peter D. Haynes, ${ }^{1}$ Chris-Kriton Skylaris,${ }^{3}$ Mike C. Payne, ${ }^{2}$ and Arash A. Mostofi ${ }^{1}$ \\ ${ }^{1}$ The Thomas Young Centre for Theory and Simulation of Materials, Imperial College London, London SW7 2AZ, United Kingdom \\ ${ }^{2}$ Theory of Condensed Matter, Cavendish Laboratory, J. J. Thomson Avenue, Cambridge CB3 OHE, United Kingdom \\ ${ }^{3}$ School of Chemistry, University of Southampton, Southampton SO17 1BJ, United Kingdom \\ (Received 3 December 2010; revised manuscript received 29 March 2011; published 2 May 2011)
}

\begin{abstract}
Linear scaling methods for density-functional theory (DFT) simulations are formulated in terms of localized orbitals in real space, rather than the delocalized eigenstates of conventional approaches. In local-orbital methods, relative to conventional DFT, desirable properties can be lost to some extent, such as the translational invariance of the total energy of a system with respect to small displacements and the smoothness of the potential-energy surface. This has repercussions for calculating accurate ionic forces and geometries. In this work we present results from ONETEP, our linear scaling method based on localized orbitals in real space. The use of psinc functions for the underlying basis set and on-the-fly optimization of the localized orbitals results in smooth potential-energy surfaces that are consistent with ionic forces calculated using the Hellmann-Feynman theorem. This enables accurate geometry optimization to be performed. Results for surface reconstructions in silicon are presented, along with three example systems demonstrating the performance of a quasi-Newton geometry optimization algorithm: an organic zwitterion, a point defect in an ionic crystal, and a semiconductor nanostructure.
\end{abstract}

DOI: 10.1103/PhysRevB.83.195102

PACS number(s): 31.15.A-, 71.15.Mb, 68.35.bg, 31.15.es

\section{INTRODUCTION}

Conventional methods for atomistic simulations based on density-functional theory ${ }^{1,2}$ (DFT), such as the plane-wave pseudopotential approach, ${ }^{3}$ have had an immense impact on the way in which material properties are studied. Their reach has extended beyond condensed-matter physics into materials science, chemistry, earth sciences, biochemistry, and biophysics. In spite of their success, the system size accessible to such techniques is limited because the algorithms scale with the cube of the number of atoms. The quest to bring to bear the predictive power of DFT calculations on ever larger systems has resulted in much interest in developing linear scaling methods for DFT simulations, ${ }^{4-14}$ and there are now a number of linear scaling DFT codes available, including ONETEP, ${ }^{15-17}$ CONQUEST, ${ }^{18,19}$ SIESTA, ${ }^{20}$ OPENMX, ${ }^{21}$ and other codes designed for large-scale simulations, such as BIGDFT $^{22}$ and FHI-AIMS. ${ }^{23}$ The ability to perform total-energy calculations in $\mathcal{O}(N)$ operations, where $N$ is the number of atoms, is only the first step toward solving real scientific problems, as most applications require structural relaxation. This means computation of the ionic forces, and as such force calculations are implemented in most of the codes listed above ${ }^{24-26}$ using a variety of choices of basis set.

One of the main advantages of using a plane-wave basis is that the basis set is independent of ionic positions, hence there are no Pulay corrections ${ }^{27}$ to the forces. As a result, the prefactor associated with calculating ionic forces is small and constitutes a negligible fraction of the total computational time. With the algorithms used in plane-wave pseudopotential (PWP) simulations, forces cost $\mathcal{O}(N)$ operations per ion, and hence $\mathcal{O}\left(N^{2}\right)$ operations in total. However, it is not immediately clear that the advantages of the PWP method for evaluation of forces can be carried over to the context of real-space linear-scaling methods, for two reasons. First, these methods must be formulated in terms of objects localized in real space, and the delocalized nature of plane waves would make them unsuitable as a basis set. Second, if one combines a basis set that is fixed in space with localization constraints on the localized functions which depend on the ion coordinates, then as the ions move, the basis functions will move relative to the localization regions and edge points may move in and out of the regions. This may result in potential-energy surfaces (PES), mapped out by displacement of the ions, that are less smooth than those obtained when the extended KohnSham orbitals of conventional DFT calculations are used. This phenomenon leads to ionic forces that are not exactly consistent with the PES, thereby limiting the accuracy and convergence rate of structural relaxations.

The linear scaling approach we address here, ONETEP, ${ }^{15}$ uses a localized basis set of psinc functions, which can be shown to be equivalent to plane waves ${ }^{28}$ and has comparable systematic convergence, overcoming the first of the difficulties listed above. In this work we investigate the effect of the second problem, namely the accuracy of ionic forces and the smoothness of the PES, and compare our results for a number of challenging cases with those obtained using conventional cubic scaling plane-wave calculations. The ionic forces have been implemented in a quasi-Newton geometry optimization scheme $^{29}$ and we show results of structural relaxation on the $\mathrm{Si}(001)$ surface and three further examples: an organic zwitterion, a point defect in alumina, and a GaAs nanocrystal. We then demonstrate the efficient scaling of these methods to very large system sizes by demonstrating the application of the method to extended DNA strands containing up to 17000 atoms.

In Sec. II the features of our method that result in its effectiveness will be discussed briefly. In Sec. III we demonstrate that, as a result of the minimization procedure used and the properties of the orthogonal psinc basis set that is employed, only the Hellmann-Feynman force on each ion is required. In Sec. IV we demonstrate the convergence and consistency of these calculated forces, in Sec. V results from 
the application of this method in the ONETEP code to realistic systems will be presented, and in Sec. VI conclusions will be drawn.

\section{THEORETICAL BACKGROUND}

Linear scaling methods exploit the "nearsightedness" 30,31 inherent in quantum many-body systems by exploiting the localization of Wannier functions ${ }^{32-35}$ or the single-particle density matrix. ${ }^{36,37}$ In ONETEP the density matrix is expressed in a separable form originally suggested by McWeeny ${ }^{38}$ and subsequently by Hernández et $a l^{9}{ }^{9}$ in the context of linear scaling calculations:

$$
\rho\left(\mathbf{r}, \mathbf{r}^{\prime}\right)=\sum_{\alpha \beta} \phi_{\alpha}(\mathbf{r}) K^{\alpha \beta} \phi_{\beta}^{*}\left(\mathbf{r}^{\prime}\right)
$$

where $\left\{K^{\alpha \beta}\right\}$ are the elements of the density kernel $^{38}$ and $\left\{\phi_{\alpha}\right\}$ are a set of atom-centered nonorthogonal generalized Wannier functions ${ }^{11}$ (NGWFs). Linear scaling is achieved by imposing spatial cutoffs for the range of the density kernel and localization radii of the NGWFs. In our procedure we mimimize the total energy with respect to both the density kernel and the NGWFs. The Brillouin zone is sampled at the $\Gamma$ point only.

In order to optimize the NGWFs they must be represented in some basis. The plane waves of conventional DFT calculations have many desirable properties: the kinetic energy operator is diagonal in momentum space; quantities are switched efficiently between real space and momentum space using fastFourier transforms; the completeness of the basis, and hence the accuracy of one's calculation, is controlled systematically with a single parameter; and, particularly relevant to this work, the ionic forces are calculated by straightforward application of the Hellmann-Feynman theorem. ${ }^{39,40}$

The extended nature of plane waves, however, would appear to make them unsuitable for describing the real-space localized orbitals used in linear scaling methods. In spite of this, ONETEP is a linear scaling method based on a plane-wave basis set that overcomes the above difficulty and is able to achieve the same accuracy ${ }^{41,42}$ and convergence rate $^{28}$ as the conventional plane-wave approach.

ONETEP uses a localized yet orthogonal basis of periodic cardinal sine (psinc) functions (defined in Ref. 28), which are formed from a discrete sum of plane waves. As such it retains many of the desirable properties inherent to the conventional plane-wave approach. The localized NGWFs that span the occupied subspace are represented in terms of these psinc functions and are optimized in situ during the calculation.

\section{IONIC FORCES AND GEOMETRY OPTIMIZATION}

In the context of Kohn-Sham DFT, the total energy $E$ is a functional of the electronic density $n(\mathbf{r})$, which is given by the diagonal part of the density matrix of Eq. (1):

$$
n(\mathbf{r})=2 \sum_{\alpha \beta} \phi_{\alpha}(\mathbf{r}) K^{\alpha \beta} \phi_{\beta}^{*}(\mathbf{r}),
$$

where the factor of 2 takes into account spin degeneracy.
In ONETEP the NGWFs are represented in terms of the underlying orthogonal psinc basis $\left\{D_{i}(\mathbf{r})\right\}$ :

$$
\phi_{\alpha}(\mathbf{r})=\sum_{i \in \operatorname{LR}(\alpha)} c_{i \alpha} D_{i}(\mathbf{r}),
$$

where $\operatorname{LR}(\alpha)$ is the spherical, atom-centered localization region of NGWF $\phi_{\alpha}$ and $\left\{c_{i \alpha}\right\}$ are its expansion coefficients in the psinc basis. Note that the localization regions move with the atoms but the locations of the points of the psinc grid are fixed in space. Overall, the total energy is variationally dependent on the coefficients $\left\{c_{i \alpha}\right\}$ and the elements $\left\{K^{\alpha \beta}\right\}$ of the density kernel. In our minimization scheme we optimize all of these degrees of freedom. ${ }^{11}$

The force on an ion at $\mathbf{R}_{\gamma}$ is given by the derivative of the total energy with respect to the ionic position,

$$
\begin{aligned}
\mathbf{F}_{\gamma}=-\frac{d E}{d \mathbf{R}_{\gamma}}= & -\frac{\partial E}{\partial \mathbf{R}_{\gamma}}-\sum_{\alpha \beta} \frac{\partial E}{\partial K^{\alpha \beta}} \frac{d K^{\alpha \beta}}{d \mathbf{R}_{\gamma}} \\
& -\sum_{\alpha} \int \frac{\delta E}{\delta \phi_{\alpha}(\mathbf{r})} \frac{d \phi_{\alpha}(\mathbf{r})}{d \mathbf{R}_{\gamma}} d^{3} r .
\end{aligned}
$$

Using Eq. (3) and the fact that the psinc basis is fixed, i.e., independent of ionic position such that $\frac{d D_{i}(\mathbf{r})}{d \mathbf{R}_{\gamma}}=0$, the last term in Eq. (4) may be expressed as

$$
\sum_{\alpha} \sum_{i \in \mathrm{LR}(\alpha)} \frac{\partial E}{\partial c_{i \alpha}} \frac{d c_{i \alpha}}{d \mathbf{R}_{\gamma}} .
$$

Although the localization regions move with the atoms, this does not have any effect on the analytic derivative $\frac{\partial E}{\partial \mathbf{R}_{\gamma}}$, because for an infinitesimal change $\delta \mathbf{R}_{\gamma}$ the set of psinc points inside the localization region, $i \in L R(\alpha)$, does not change.

At the end of the electronic minimization, if we can assume that the total energy is at a minimum with respect to the degrees of freedom of the density, then we will satisfy the conditions

$$
\frac{\partial E}{\partial K^{\alpha \beta}}=0 \text { and } \frac{\partial E}{\partial c_{i \alpha}}=0,
$$

and we are on the Born-Oppenheimer surface for the given ionic configuration. Under these conditions, the second and third terms in Eq. (4) vanish, leaving only the HellmannFeynman force

$$
\mathbf{F}_{\gamma}=-\frac{\partial E}{\partial \mathbf{R}_{\gamma}},
$$

which can be calculated in much the same spirit as the components of the total energy itself, using our "fast-Fourier transform (FFT) box" technique ${ }^{43,44}$ to switch quantities efficiently between real and reciprocal space.

The only components of the total energy with an explicit dependence on $\mathbf{R}_{\gamma}$ are the ion-ion and electron-ion terms. With nonlocal ionic pseudopotentials, there are both local and nonlocal contributions to the latter. Written in KleinmanBylander form, ${ }^{45}$ the nonlocal pseudopotential energy is given by

$$
E_{\mathrm{nl}}=\sum_{\alpha \beta} \sum_{i}\left\langle\phi_{\alpha} \mid \chi_{i}\right\rangle D_{i}\left\langle\chi_{i} \mid \phi_{\beta}\right\rangle K^{\beta \alpha},
$$

where $\chi_{i}$ is the $i$ th projector, the sum over $i$ runs over all the projectors on all atoms, and $D_{i}$ is its Kleinman-Bylander 
energy. The nonlocal contribution to the force on atom $\gamma$ is then

$$
\begin{aligned}
\mathbf{F}_{\gamma}^{\mathrm{nl}=} & -\frac{\partial E_{\mathrm{nl}}}{\partial \mathbf{R}_{\gamma}}=-\sum_{\alpha \beta} \sum_{i} \\
& \left.\times\left[\left\langle\phi_{\alpha} \mid \frac{\partial \chi_{i}}{\partial \mathbf{R}_{\gamma}}\right\rangle D_{i}\left\langle\chi_{i} \mid \phi_{\beta}\right\rangle-\left\langle\phi_{\alpha} \mid \chi_{i}\right\rangle D_{i}\left|\frac{\partial \chi_{i}}{\partial \mathbf{R}_{\gamma}}\right| \phi_{\beta}\right\rangle\right] K^{\beta \alpha},
\end{aligned}
$$

where the sum over projectors runs only over those projectors on atom $\gamma$. Since the projectors are only nonzero within the core region of each ion and the NGWFs are strictly localized, projector-NGWF overlap matrices $\left\langle\chi_{i} \mid \phi_{\beta}\right\rangle$ are highly sparse, and evaluation of the overlaps is performed within the "FFT box" approximation. ${ }^{43,44}$ The nonlocal contribution to the energy and all ionic forces can therefore be calculated in $O(N)$ computational effort.

For the long-ranged Coulombic ion-ion and electron-ion terms, there are ways to reformulate the Ewald method so that they scales as $O(N \ln N)$ with suitable approximations involving transferring the point charges to a grid. ${ }^{46}$ These methods are routinely employed in classical molecular dynamics (MD) codes, but are not easily amenable to high-accuracy $O(N)$ methods in the size range considered here and come at a cost in accuracy. Fortunately, the evaluation of these terms is nonetheless computationally straightforward: within the Ewald approach, the ion-ion term is

$$
\mathbf{F}_{\gamma}^{\mathrm{ew}}=-\frac{\partial E_{\mathrm{ew}}}{\partial \mathbf{R}_{\gamma}}
$$

which can be evaluated easily by standard techniques and can be made to scale as $O\left(N^{3 / 2}\right)$ or better, ${ }^{47}$ with suitably chosen parameters. The local ionic pseudopotential contribution is most easily evaluated in reciprocal space, as

$$
\mathbf{F}_{\gamma}^{\mathrm{loc}}=-\frac{\partial E_{\mathrm{loc}}}{\partial \mathbf{R}_{\gamma}}=\sum_{\mathbf{G}} i \mathbf{G} e^{-i \mathbf{G} \cdot \mathbf{R}_{\gamma}} V_{\gamma}^{\mathrm{loc}}(\mathbf{G}) n^{*}(\mathbf{G}),
$$

where $V_{\gamma}^{\text {loc }}$ is the local pseudopotential of atom $\gamma$. This is also relatively straightforward to compute but clearly asymptotically involves $O\left(N^{2}\right)$ computational effort in this formulation, since the number of $\mathbf{G}$ vectors in the simulation cell scales as $O(N)$ and the summation must be performed for all $N$ atoms. As for the Ewald approach, it is possible to reformulate this as an $O(N \ln N)$ algorithm, ${ }^{48,49}$ but as will be seen in Sec. IV, the $O\left(N^{2}\right)$ contribution to the forces calculation has a small prefactor compared to the $O(N)$ evaluation of the total energy, and does not become problematic until the very largest system sizes currently encountered in linear-scaling DFT calculations. To go further, one could alternatively use fast multipole methods to reduce the scaling. ${ }^{50}$

Finally, in systems with nonlinear core corrections to the exchange-correlation energy, ${ }^{51,52}$ there is an additional contribution to the force due to the fact that the core density moves with the ion. This is also most easily evaluated in reciprocal space, with a similar prefactor to the local potential term:

$$
\mathbf{F}_{\gamma}^{\mathrm{nlcc}}=-\frac{\partial E_{\mathrm{nlcc}}}{\partial \mathbf{R}_{\gamma}}=\sum_{\mathbf{G}} i \mathbf{G} e^{-i \mathbf{G} \cdot \mathbf{R}_{\gamma}} n_{\gamma}^{\mathrm{c}}(\mathbf{G}) V_{\mathrm{xc}}^{*}(\mathbf{G}) .
$$

Equation (7) is correct in the limit in which no localization constraints are imposed on the NGWFs. With localization constraints, the translational invariance of the system with respect to the grid of psinc basis functions is broken, ${ }^{53}$ coined the "egg-box" effect, ${ }^{54}$ which introduces an error in the force that is, in general, difficult to calculate explicitly but which may be controlled by decreasing the grid spacing or increasing the radius of the localization regions. ${ }^{55}$ This phenomenon is related to the fact that the underlying basis of psinc functions is fixed with respect to the ions while the LRs are atom-centered and therefore move with the atoms, resulting in each NGWF having a nonequivalent representation in the psinc basis depending on its exact position with respect to the grid of psinc functions. As will be demonstrated in Sec. IV, forces calculated in ONETEP according to Eq. (7) are already very accurate, even for weakly bonded systems, and have been implemented in a quasi-Newton geometry optimization scheme $^{56}$ based on the Broyden-Fletcher-Goldfarb-Shanno (BFGS) algorithm (see, e.g., Ref. 57).

\section{PRELIMINARY TESTS}

\section{A. Convergence in molecular systems}

We start with preliminary calculations to examine issues of calculating individual forces. First we present two very simple, small-scale test cases: (i) a symmetric stretch of a carbon dioxide molecule, and (ii) a hydrogen bond in a water dimer. In order to demonstrate the accuracy of potential-energy surfaces obtained from ONETEP, we compare with equivalent calculations with the CASTEP $^{29}$ plane-wave pseudopotential code. In all comparisons we use identical norm-conserving pseudopotentials ${ }^{58}$ in Kleinman-Bylander separable form, ${ }^{45}$ the same local-density approximation ${ }^{59}$ for the exchange-correlation functional, and $\Gamma$-point sampling of the Brillouin zone.

We must first investigate the convergence of calculated quantities with respect to the basis size in the two methods. In ONETEP we can systematically control the convergence by decreasing the grid spacing (which corresponds to a plane-wave cutoff $E_{c}$ ) and increasing the localization radii $R_{\phi}$ of the NGWFs. In CASTEP we can vary the plane-wave cutoff $E_{c}$. In Fig. 1 we examine the convergence of the total energy of the two molecular systems with respect to these quantities, while in Fig. 2 we examine the convergence of force components. It is observed, as previously noted, ${ }^{60}$ that total-energy convergence with grid spacing is slower in ONETEP than in plane-wave methods such CASTEP. This is due to the greater influence of the so-called "egg-box" effect in the former (the variation in energy with uniform translation of the atoms with respect to the grid), which results from NGWF truncation to points within a sphere on a regular underlying grid. However, both methods converge asymptotically to the same value to a high precision. The ONETEP forces converge nonmonotonically with both $E_{c}$ and $R_{\phi}$ to eventual good agreement with the CASTEP equivalents, at a radius not significantly greater than would be required for tolerable convergence of the total energy (around $4.0 \AA$ in this case). It is to be noted that at a fixed, underconverged value of $E_{c}$, convergence with $R_{\phi}$ is to an incorrect value, while at fixed, underconverged $R_{\phi}$, convergence with $E_{c}$ may be erratic or tend to an incorrect 

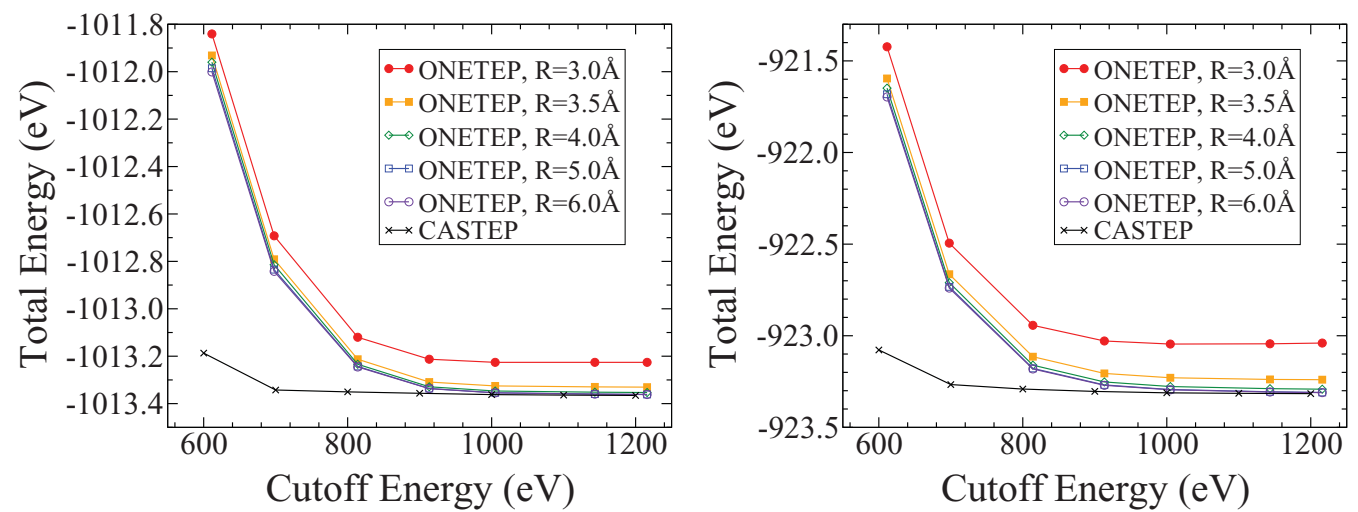

FIG. 1. (Color online) (Color online) Total energy as calculated by ONETEP and CASTEP at a fixed geometry as a function of cutoff energy $E_{c}$ and NGWF truncation radius $R_{\phi}$, for the $\mathrm{CO}_{2}$ molecule (left) and an $\mathrm{H}_{2} \mathrm{O}$ dimer (right). Convergence properties are dominated by the oxygen pseudopotential and so are broadly the same for the two systems. The results for $4.0-\AA$ spheres to $6.0-\AA$ spheres agree to high accuracy, particularly at a cutoff energy of $900 \mathrm{eV}$ or above, indicating convergence at around $4.0 \AA$.

value. In particular, very small localization regions result in highly inaccurate forces. This emphasizes the importance of converging with respect to both parameters simultaneously for accurate results. Finally, as with for plane-wave codes, it should be noted that accurate forces will often require higher convergence parameters than accurate energy differences.

To demonstrate that the convergence properties demonstrated here are applicable to larger systems, we examine the difference between the calculated forces in ONETEP and CASTEP for a larger molecule. We employ the organic zwitterionic detergent molecule, 3-[(3Cholamidopropyl)dimethylammonio]-1-propanesulfonate, or "CHAPS" as a particularly challenging, worst-case test system, due to the considerable charge separation and long-ranged forces encountered. In Fig. 3 we plot the rms error of all the calculated forces, and observe that they can be systematically converged with respect to local orbital radius and cutoff energy.

\section{B. Consistency in molecular systems}

We now examine the consistency of the forces and the energy by calculating the full binding curve for the $\mathrm{CO}_{2}$ molecule and the $\mathrm{H}_{2} \mathrm{O}$ molecular dimer. For the CASTEP calculation a plane-wave energy cutoff energy of $1100 \mathrm{eV}$ was used for the wave functions, while for ONETEP a grid spacing of $0.227 \AA$ (equivalent to a plane-wave energy cutoff of $1121 \mathrm{eV}$ ) and four NGWFs on each atom, each with a localization radius of $4.0 \AA$, were used. Examination of Figs. 1 and 2 suggest these cutoffs will produce results accurate to within around $10 \mathrm{meV}$ for the total energy and $0.01 \mathrm{eV} / \AA$ for the forces in these two systems.

In Fig. 4 the variation of the total energy of a carbon dioxide molecule with respect to the $\mathrm{C}-\mathrm{O}$ bond length is shown, as calculated with CASTEP (plus symbols) and ONETEP (open diamonds). The curve drawn is a polynomial fit $E(x)$ to the data points. As can be seen, the results are indistinguishable. The inset to Fig. 4 shows the Hellmann-Feynman force, calculated according to Eq. (7), in CASTEP (plus symbols) and ONETEP (open diamonds). Again the agreement is excellent. The curve shown in the inset is the analytic derivative $F(x) \equiv-\frac{1}{2} \frac{d E}{d x}$ of the polynomial fit to the energy data points. A discrepancy between this curve and the calculated forces would indicate an inconsistency between the PES and the Hellmann-Feynman forces. We see that there is no inconsistency and that any
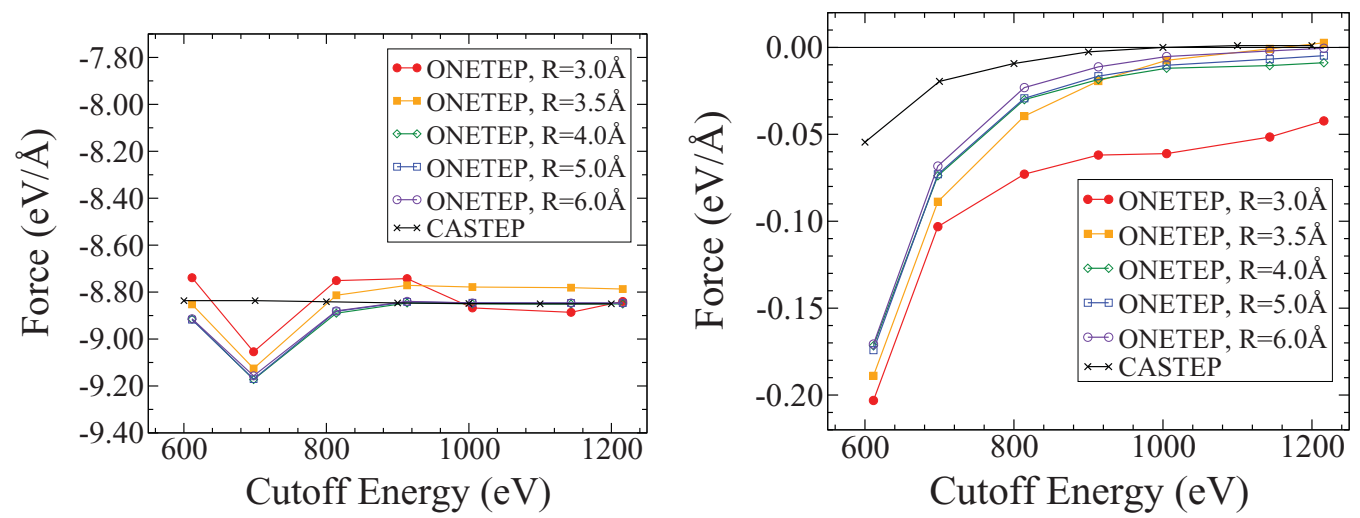

FIG. 2. (Color online) Convergence properties of a single force component calculated using ONETEP and CASTEP at a fixed, off-equilibrium geometry as a function of cutoff energy $E_{c}$ and NGWF truncation radius $R_{\phi}$, for the $\mathrm{CO}_{2}$ molecule (left) and an $\mathrm{H}_{2} \mathrm{O}$ dimer (right). Force shown is the force acting on an $\mathrm{O}$ atom along the bond axis for $\mathrm{CO}_{2}$ (hence a very strong force), and the force on the hydrogen atom not involved in the dimer-dimer $h$ bond (hence a very weak force, near equilibrium). Forces converge with both $R_{\phi}$ and $E_{c}$, but care must be taken to accurately converge with respect to both quantities simultaneously. 


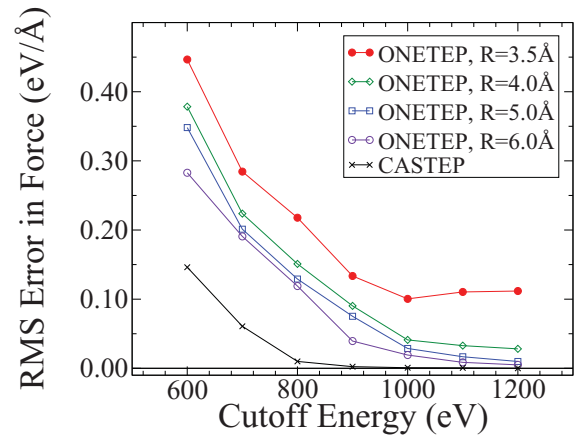

FIG. 3. (Color online) Convergence properties of the RMS error in the ONETEP and CASTEP forces at a fixed, off-equilibrium geometry as a function of cutoff energy $E_{c}$ and NGWF truncation radius $R_{\phi}$, for the CHAPS molecule (see text). The quantity plotted is the RMS difference between the calculated result for a given $E_{c}$ and $R_{\phi}$ and the calculated result for $E_{c}=1200 \mathrm{eV}$ in CASTEP, which is taken to be the converged result. We see that with respect to both $R_{\phi}$ and $E_{c}$ it is possible to obtain systematic convergence to the plane-wave result.

errors introduced by imposing localization constraints on the NGWFs are negligible. Quantitative comparison of the two approaches shows that the fractional differences in the equilibrium bond length and the curvature of the PES at the minimum, respectively, are less than $0.1 \%$ and $0.2 \%$. The discrepancy between the equilibrium bond length as predicted by the numerical force [given by the $x$ intercept of $F(x)$ ] and by the Hellmann-Feynman force (given by a polynomial fit to the force data points) is less than $0.1 \%$ for both approaches.

We turn now to the more challenging case of the water dimer. In Fig. 5 the total energy as a function of the length of the hydrogen bond is shown. This is a much more sensitive test, as the potential well associated with the hydrogen bond is

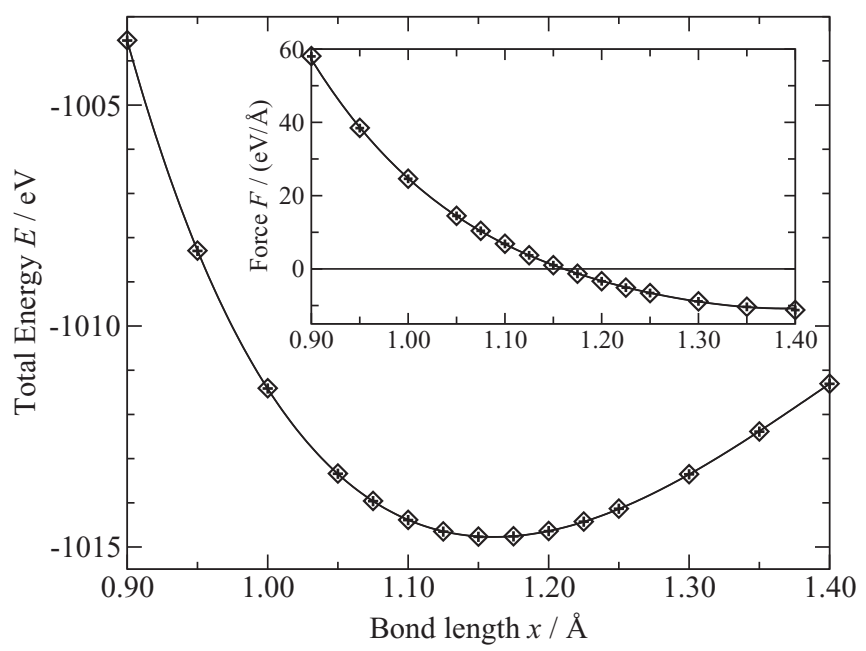

FIG. 4. Total energy as a function of C-O bond-length for a carbon dioxide molecule subjected to a symmetric stretch. CASTEP (plus symbols); ONETEP (open diamonds). The curve shows a polynomial fit $E(x)$ to the data points. On the scale of this plot the data are indistinguishable. Inset: the Hellmann-Feynman force on each oxygen atom. The curve shows the numerical force $F(x) \equiv-\frac{1}{2} \frac{d E}{d x}$.

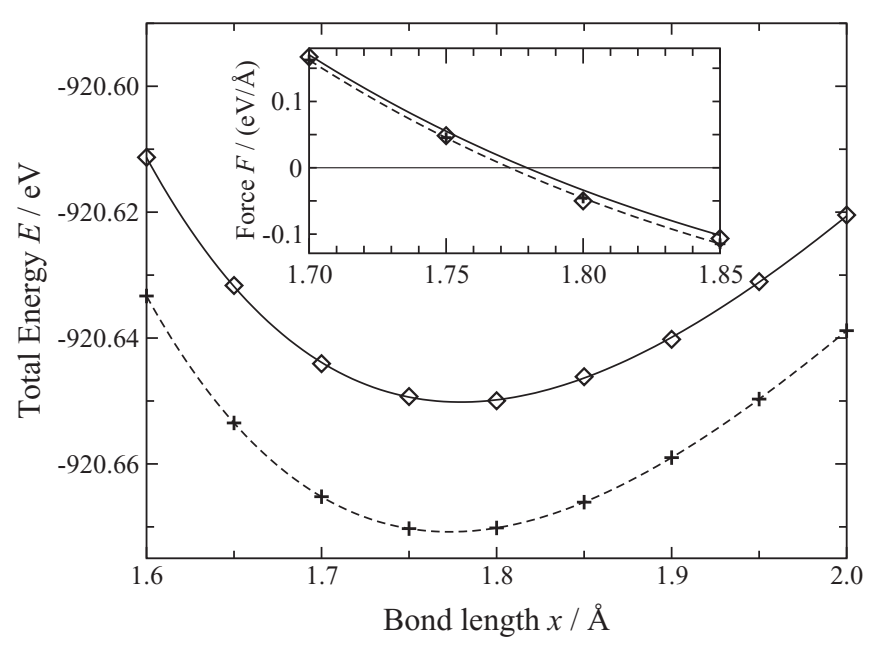

FIG. 5. Interaction potential of a water dimer. CASTEP (plus symbols); ONETEP (open diamonds). The curves show polynomial fits $E(x)$ to the data points. Inset: the Hellmann-Feynman force on each water molecule. The curves show the numerical force $F(x) \equiv-\frac{d E}{d x}$ for CASTEP (dashed line) and ONETEP (solid line).

shallower and the forces weaker by two orders of magnitude than in the case of the strong covalent bonds in carbon dioxide.

For the CASTEP calculation a plane-wave cutoff energy of $1200 \mathrm{eV}$ was used. For ONETEP, a grid spacing of $0.214 \AA$ (equivalent to a plane-wave energy cut-off of $1261 \mathrm{eV}$ ) was used; each hydrogen and oxygen atom had one and four NGWFs, respectively, all of radius $4.0 \AA$.

From Fig. 5 it may be seen that the total energies of the two approaches are within $20 \mathrm{meV}$ of each other. The predicted equilibrium bond length and the curvature of the PES at the minimum agree to within $0.3 \%$ and $4.6 \%$, respectively, well within the variations associated with using different exchange and correlation functionals. The effect of localization constraints, however, is now apparent. The inset shows that the Hellmann-Feynman forces in ONETEP do not coincide perfectly with the derivative of the fit to the total energy. Nevertheless, the discrepancy is small: at the equilibrium bond length the error, defined as the fractional difference between the $x$ intercept of the numerical force (solid line, inset of Fig. 5) and that of a fit (not shown) to the Hellmann-Feynman forces (open diamonds, inset of Fig. 5) is only $0.3 \%$, or $0.005 \AA$. Note that the forces in ONETEP agree with the forces in CASTEP even more accurately than the derivative of the fit to the ONETEP total energy agrees with the corresponding forces. This correlates well with the observed insensitivity of the calculated forces to the effect of truncation of the local orbitals.

\section{Convergence and consistency in bulk systems}

The above tests for molecules demonstrate the basic applicability of the methods for evaluation of forces within the context of this particular local-orbital method, but do not test their accuracy in the more challenging conditions of a solid, with the constraints necessary for linear scaling. In particular, in a solid, convergence with local orbital radius can present greater difficulties. To demonstrate the convergence 
behavior in solids, we simulate a block of bulk silicon (diamond structure) subject to random distortions of the atomic coordinates about their equilibrium positions and compare the calculated ONETEP forces on these displaced atoms with those calculated in CASTEP.

We begin with a supercell consisting of $5 \times 5 \times 5$ times the 8-atom cubic unit cell of the bulk silicon with lattice parameter $a=5.4 \AA$, giving 1000 atoms. We then generate a set of realizations of random disorder by displacing each atom according to a uniform random distribution with a given amplitude. These systems are not intended to be physically meaningful apart from as a test of the accuracy of the calculated forces, but are approximately representative of a snapshot of the system at elevated temperature. Calculations were performed with ONETEP for $R_{\phi}=4.23 \AA, R_{\phi}=4.76 \AA$, and $R_{\phi}=5.29 \AA$. A fixed psinc spacing of $0.256 \AA$ corresponding to a plane-wave cutoff of $E_{c}=883 \mathrm{eV}$, which was verified to give good convergence of both total energies and forces in CASTEP, was used in both codes. In the ONETEP calculations, a kernel cutoff greater than the supercell size was employed, meaning all elements of the density kernel were nonzero, and optimized using the Li-Nunes-Vanderbilt (LNV) energy minimization scheme. ${ }^{7}$ The CASTEP calculations were performed on identical 1000-atom cells with the same plane-wave cutoff. We employed the local-density approximation for exchange and correlation. For many systems it is possible to obtain plane-wave accuracy using only as many NGWFs as valence orbitals. ${ }^{60}$ However, previous ONETEP calculations on bulk silicon $^{61}$ have reported that nine NGWFs per silicon atom are required to achieve plane-wave accuracy, and this prescription was followed here.

Table I shows the convergence of the forces with respect to the local orbital radius. We see that by $R_{\phi}=4.23 \AA$ results are already in reasonable agreement with equivalent plane-wave results, with an RMS deviation increasing from $0.001 \mathrm{eV} / \AA$ to $0.004 \mathrm{eV} / \AA$ as the disorder magnitude $\Delta$ increases. For larger radii the results improve, though the extent to which they can agree with the CASTEP results is limited by the egg-box effect resulting from the underlying grid spacing. By $\Delta=0.5 a_{0}$, the system is some way off equilibrium, with an rms force of around $2.3 \mathrm{eV} / \AA$, but the precision of the agreement with plane-wave results is maintained.

\section{APPLICATIONS}

\section{A. Si surface reconstructions}

We now report results of a realistic application of geometry optimization, using our quasi-Newton scheme, on a $\mathrm{Si}(001)$ surface, comparing again with CASTEP. Calculations were performed within the local-density approximation in a $8 \times 8$ supercell consisting of nine atomic layers of silicon atoms in which the bottom layer of atoms was hydrogen passivated (a total of 640 atoms). A fixed lattice constant of $5.43 \AA$ was used, resulting in a supercell of dimensions $30.713 \AA \times 30.713 \AA$ in the plane of the surface. The size of the supercell in the perpendicular direction was $25.595 \AA$, providing a vacuum gap of $12.9 \AA$ between adjacent periodic replicas, though this varies slightly during the calculation. For these surface calculations, the same grid spacing of $0.256 \AA$ was used, and localization radii of $4.0 \AA$ were chosen.

The passivating hydrogen atoms were constrained to lie vertically below the bottom layer of silicon atoms which were fixed to their bulk positions. Surface atoms were given small initial random displacements to break symmetry so that they could dimerize. Symmetry was imposed so that the surface could only form a $p(2 \times 1)$ reconstruction. This allows a direct comparison with a CASTEP calculation on a $2 \times 1$ surface supercell comprised of 18 silicon atoms and two passivating hydrogen atoms. A plane-wave cutoff of $883 \mathrm{eV}$ was used for the wave functions and the Brillouin zone was sampled using an evenly spaced grid consisting of $4 \times 8 k$ points in CASTEP. The same pseudopotentials were used for both ONETEP and CASTEP calculations.

As expected, surface atoms were observed to pair up to form dimers, which then buckled out of the plane of the surface. The resulting geometry is shown in Fig. 6. Bond lengths and buckling angles are compared in Table II. They compare very well with all bond lengths lying within $0.02 \AA$. The differences in bond lengths lead to a slightly smaller buckling angle in CASTEP compared to ONETEP. The bond lengths also compare well with those found in previous work by Ramstad et al. ${ }^{62}$ The shorter bond lengths found in that work can be attributed to the use of different pseudopotentials.

Regarding the number of NGWFs/atom, we observed here that using only four NGWFs per silicon, surface atoms

TABLE I. Convergence with NGWF radius of atomic forces $(\mathrm{eV} / \AA ̊$ ) for a 1000 -atom system of bulk Si subject to random displacements of $\Delta=\{0,0.05,0.5\} a_{0}$, where $a_{0}=0.529 \AA . \mathbf{F}$ is the force calculated in ONETEP and $\mathbf{F}_{\mathrm{CAS}}$ its equivalent calculated in CASTEP. Maximum and root-mean-square forces, plus corresponding values for the maximum and rms deviation of forces from the CASTEP result are shown for each of $R_{\phi}=\{8,9,10\} a_{0}$. Note that for $\Delta=0$, the CASTEP forces are all zero by symmetry, and the deviation of the ONETEP results is solely due to the egg-box effect and the influence of NGWF truncation.

\begin{tabular}{lccccc}
\hline \hline$\Delta\left(a_{0}\right)$ & $R_{\phi}\left(a_{0}\right)$ & $|\mathbf{F}|_{\max }$ & $\left|\mathbf{F}-\mathbf{F}_{\text {CAS }}\right|_{\max }$ & $|\mathbf{F}|_{\text {rms }}$ & $\left|\mathbf{F}-\mathbf{F}_{\text {CAS }}\right|_{\text {rms }}$ \\
\hline 0.00 & 8.0 & 0.00135 & 0.00135 & 0.00096 & 0.00096 \\
0.00 & 9.0 & 0.00020 & 0.00020 & 0.00014 & 0.00014 \\
0.00 & 10.0 & 0.00011 & 0.00011 & 0.22083 & 0.00008 \\
0.05 & 8.0 & 0.40748 & 0.00437 & 0.22095 & 0.00193 \\
0.05 & 9.0 & 0.40772 & 0.00357 & 0.22091 & 0.00175 \\
0.05 & 10.0 & 0.40779 & 0.00354 & 2.22984 & 0.00169 \\
0.50 & 8.0 & 6.1615 & 0.00870 & 2.30108 & 0.00208 \\
0.50 & 9.0 & 6.1648 & 0.00450 & 2.29999 & 0.00270 \\
0.50 & 10.0 & 6.1620 & 0.00739 & & \\
\hline \hline
\end{tabular}




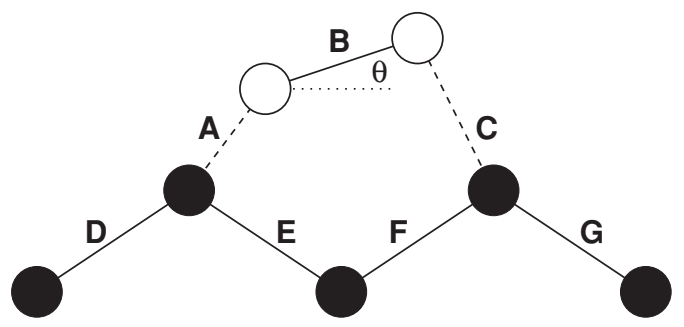

FIG. 6. Schematic of the reconstructed $\mathrm{Si}(001)$ surface. Bonds indicated by dashed lines do not lie in the plane of the diagram. The surface atoms (indicated by white circles) pair up to form dimers, that then buckle out of the plane of the surface. For the purposes of this work the bonds have been labeled alphabetically and the buckling angle denoted by $\theta$.

did dimerize but the resulting dimers failed to buckle. The flexibility afforded by nine NGWFs appears to be required for the dimers to relax into the buckled geometry.

\section{B. Charge redistribution}

Finally we report on geometry convergence tests for structural optimization in more complex systems exhibiting charge redistribution, to test the performance of the algorithms. System (a) is the CHAPS molecule referred to in Sec. IV A. Its zwitterionic nature leads to considerable charge separation and some relatively long-ranged contributions to the forces, hence a challenging case for geometry relaxation. We started from standard crystallographic data, ${ }^{63}$ with hydrogen atoms added to saturate dangling bonds. The resulting molecule contains 100 atoms. System (b) is a crystalline ceramic of 119 atoms, comprising a $2 \times 2 \times 1$ supercell of $\alpha$-alumina in the corundum structure, containing one aluminium vacancy $V_{\mathrm{Al}}{ }^{-3}$ in charge state -3 (such that neighboring oxygens retain filled $p$ shells). The starting configuration used for the relaxation was the optimized bulk geometry before removal of the aluminium atom. In this ionic system, containing a vacancy with a large net charge, there are again considerable long-ranged relaxations. Finally, system (c) is a small nanocrystal of wurtize structure GaAs, a polar semiconductor. Starting from the bulk wurtzite crystal structure optimized within DFT, the nanocrystal is imagined to have been formed by cleaving to expose [0001] faces on the two ends, corresponding to Ga and As layers,
TABLE II. Bond lengths (in $\AA$ ) and the buckling angle $\theta$ as calculated by ONETEP, CASTEP, and Ramstad et al. (Ref. 62).

\begin{tabular}{llccccccc}
\hline \hline & \multicolumn{1}{c}{$\mathrm{A}$} & $\mathrm{B}$ & $\mathrm{C}$ & $\mathrm{D}$ & $\mathrm{E}$ & \multicolumn{1}{c}{$\mathrm{F}$} & $\mathrm{G}$ & $\theta$ \\
\hline ONETEP & 2.306 & 2.272 & 2.364 & 2.362 & 2.398 & 2.333 & 2.364 & $17.9^{\circ}$ \\
CASTEP & 2.313 & 2.274 & 2.371 & 2.378 & 2.403 & 2.338 & 2.380 & $17.0^{\circ}$ \\
Ref. 62. & 2.29 & 2.26 & 2.34 & 2.35 & 2.38 & 2.33 & 2.35 & $18.3^{\circ}$ \\
\hline \hline
\end{tabular}

respectively. There remains a net dipole moment parallel to the $c$ axis, whose value depends on the geometry of the surfaces. The rod, comprising 204 atoms once dangling bonds are terminated with hydrogen, was simulated inside a cubic simulation cell of side length $45 \AA$. These systems are illustrated in Fig. 7.

Figure 8 shows the convergence behavior of the maximum force as the BFGS algorithm progresses in each case. In all three cases, convergence is achieved after 20 to 30 iterations. The forces agree to good precision with those obtained in comparable calculations in CASTEP, so the optimizations follow a similar path. The demands of convergence tolerance on plane-wave cutoff and sizes of the localization regions are not significantly greater than those required for accurate evaluation of the energy in these systems. As with plane-wave calculations, tight convergence of the electronic energy is required before the forces are well converged, since the error in the forces scales approximately as the square root of the error in the energy. We therefore conclude that it is possible to perform geometry optimization in the current framework with a similar relative performance overhead compared to single-point energies as in plane-wave DFT.

\section{Scaling with system size}

Finally, we demonstrate the scaling of the timings of the evaluation of the forces compared to the total-energy minimization. As we have described, the efficient parallel algorithms used ensure that despite the $O\left(N^{2}\right)$ prefactor on parts of the force calculation, the total computational time remains dominated by optimization of the NGWFs and density kernel at each BFGS trial step up to very large $N$. We show in Fig. 9 the total time taken by various parts of the calculation for a series of systems each comprising double helices of DNA of increasing length (with randomly chosen base pair sequences
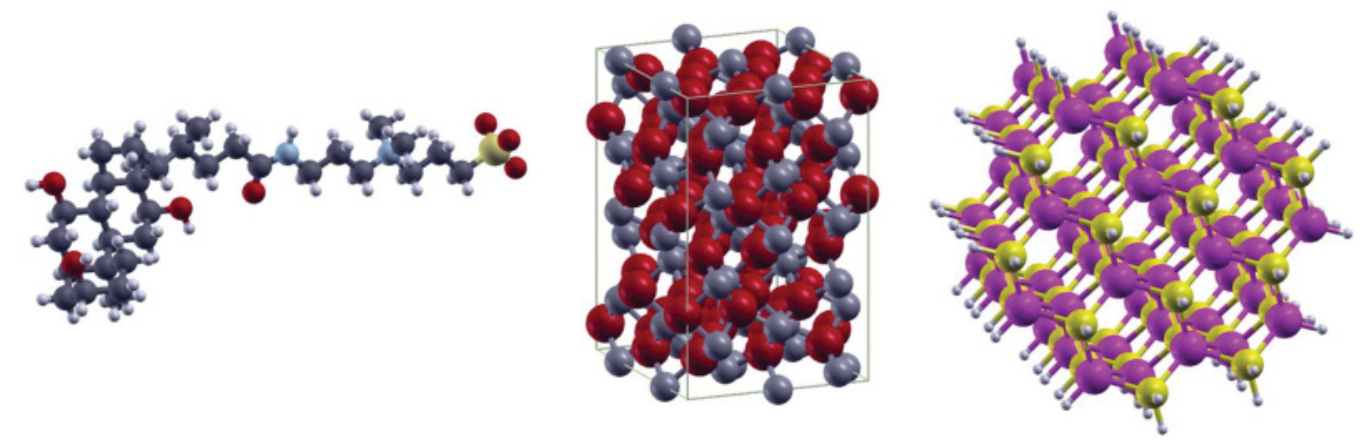

FIG. 7. (Color online) Systems for which geometry optimization was performed with the BFGS algorithm in ONETEP for illustration of convergence behavior. Left: CHAPS molecule (100 atoms) Center: Al vacancy in $2 \times 2 \times 1$ supercell of $\alpha$-alumina (119 atoms) Right: H-terminated GaAs nanocrystal (204 atoms). 


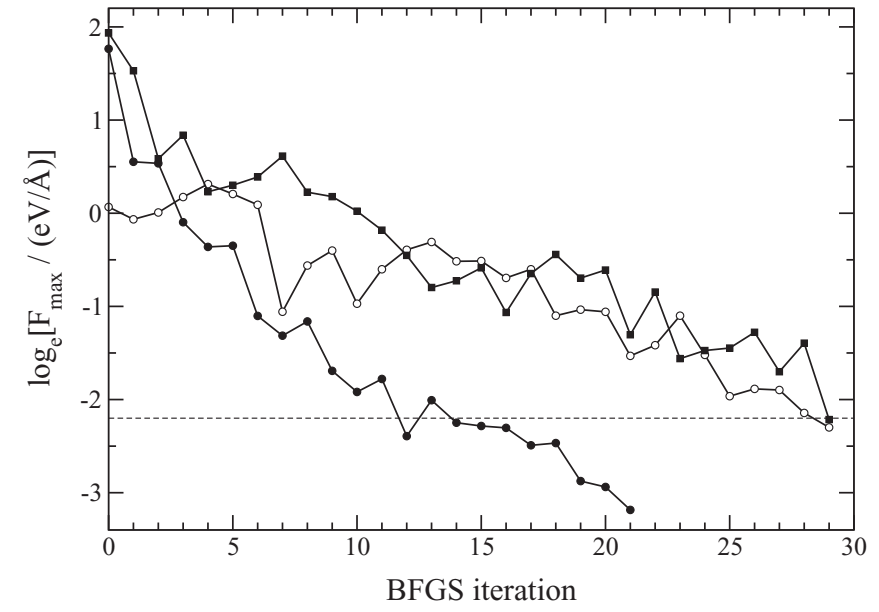

FIG. 8. Convergence behavior of the maximum force $\left|F_{\max }\right|$ on any atom as the BFGS algorithm proceeds for the typical systems shown in Fig. 7. CHAPS Zwitterion (open circles); Al vacancy in alumina (filled circles); GaAs nanocrystal (squares). Dashed line shows convergence threshold of $0.11 \mathrm{eV} / \AA$. Simultaneous convergence of total energy and the maximum displacement were required, with $\left|d \mathbf{R}_{\max }\right|=0.002 \AA$ for 2 successive iterations was also required. From the starting coordinates (see text) convergence was achieved in 29, 21, and 29 iterations, respectively.

to ensure no advantage can be gained through periodicity). The base-pair sequences were generated randomly, and the atom positions created with the Nucleic Acid Builder ${ }^{64}$ code. The positions were relaxed within an empirical potential framework, using the Amber code. ${ }^{65}$ This generated a starting point where the forces on the atoms were low but nonzero, since the empirical-potential forces do not exactly match those from the (presumably more accurate) DFT calculation.

This system has previously been shown to exhibit good linear scaling with number of atoms $N$, and scale well to large numbers of processors. ${ }^{66}$ The calculations here were performed with a plane-wave energy cutoff of $700 \mathrm{eV}$, localization radii $3.7 \AA$, and a density kernel cutoff radius of $16 \AA$. These are somewhat lower accuracy values than used in the previous tests, so as to allow scaling to very large system sizes within moderate memory requirements, but should still allow for reasonable convergence of the forces according to the findings in Sec. IV A. Note that in DNA, with a very small highest occupied molecular orbital-lowest unoccupied molecular orbital gap, the density matrix is quite long-ranged and a relatively large cutoff must be used.

We report in Fig. 9 timings for a total-energy minimization followed by a calculation of the forces on 256 parallel cores (Intel COREi7 CPUs). We vary the size of the system from 1042 atoms ( 16 base pairs) up to 16775 atoms (256 base pairs), scaling the unit cell commensurately along one direction. Note that even the smallest of these systems would be beyond the feasible scope of conventional PWP methods, given the size of the simulation cell. The total time for the optimization of the electronic degrees of freedom is seen to scale nearly perfectly as $O(N)$, while the calculation of the local pseudopotential forces scales as roughly $O\left(N^{2}\right)$ (though the improved computational load balance at large system sizes masks this slightly). Consequently, the fraction of the total time accounted for by the

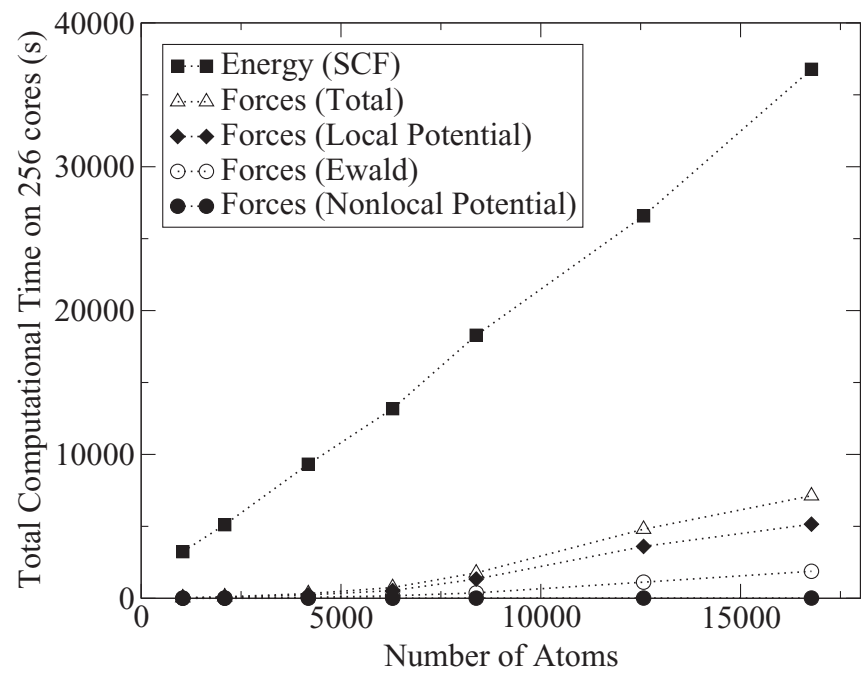

FIG. 9. Scaling of total computational time for SCF total-energy calculation for a series of DNA molecules of increasing length (squares), compared to scaling of total computational time for forces calculation (triangles), and the three force components present: Ewald, local potential, and nonlocal potential (diamonds, empty circles, and filled circles, respectively), for the same simulations.

forces increases from under $1 \%$ to nearly $20 \%$. Eventually, the calculation of forces would dominate and the method could no longer be termed linear scaling. However, this is not expected to be the case in typical systems such as the DNA strands shown here until upward of 30000 atoms. Of course, this does not imply that a fully converged geometry optimization would be necessarily possible in linear-scaling computational effort. An additional problem is the fact that ionic relaxation requires a number of iterations, or evaluations of the potential-energy surface (PES), which increases with the number of atoms. ${ }^{67}$ Preliminary steps have been taken by other authors ${ }^{67-70}$ toward addressing this issue in the context of large-scale calculations. This is outside the scope of the present work, however; often in practice it will be sufficient to relax a smaller subregion of a very large system, thereby making the optimization procedure tractable, or one might in any case be investigating the effect of a localized perturbation to an otherwise relaxed system. In such cases, we have shown that geometry optimization with plane-wave accuracy and linear-scaling computational effort is achievable up to tens of thousands of atoms.

\section{CONCLUSIONS}

In conclusion, we have shown that the combination of using strictly localized nonorthogonal generalized Wannier functions, which move with the ions but that are optimized on the fly within a basis set of psinc functions that are fixed with respect to the ions, within the ONETEP linear scaling DFT method, results in potential-energy surfaces that are sufficiently smooth that ionic forces can be calculated with high accuracy. We have demonstrated that these forces can be systematically converged with respect to energy cutoff and local orbital radius to high precision with low overhead relative to the demands of a comparable total-energy calculation. We have demonstrated this by performing geometry optimization 
on a set of widely varied systems and comparing to calculations using conventional plane-wave DFT. We note that for weaker bonds, such as hydrogen bonds, although the discrepancy between the PES and the calculated forces becomes more noticeable (Fig. 5), the effect is nonetheless very small. Finally, we have demonstrated that geometry optimization is possible with a comparable computational overhead to that for PWP simulations, and that the forces calculation, while scaling as $\mathcal{O}\left(N^{2}\right)$, remains a small fraction of the total computational time until upward of 30000 atoms for typical systems.

\section{ACKNOWLEDGMENTS}

We would like to thank Matt Probert for use of his BFGS algorithm. The authors acknowledge the support of the Engineering and Physical Sciences Research Council EPSRC (Grant No. EP/G055882/1) for funding through the HPC Software Development program. P.D.H. and C.-K.S. acknowledge the support of University Research Fellowships from the Royal Society. A.A.M. acknowledges the support of the RCUK fellowship program. The authors are grateful for the computing resources provided by Imperial College's High Performance Computing services CX1 and CX2, which have enabled most of the simulations presented here. This work also made use of the facilities of HECToR, the UK's national high-performance computing service, for some of the larger calculations. HECToR was provided by UoE HPCx Ltd at the University of Edinburgh, Cray Inc and NAG Ltd, and funded by the Office of Science and Technology through EPSRC's High End Computing Programme. *nicholas.hine@imperial.ac.uk

${ }^{1}$ P. Hohenberg and W. Kohn, Phys. Rev. 136, B864 (1964).

${ }^{2}$ W. Kohn and L. J. Sham, Phys. Rev. 140, A1133 (1965).

${ }^{3}$ M. C. Payne, M. P. Teter, D. C. Allan, T. A. Arias, and J. D. Joannopoulos, Rev. Mod. Phys. 64, 1045 (1992).

${ }^{4}$ S. Goedecker, Rev. Mod. Phys. 71, 1085 (1999).

${ }^{5}$ W. T. Yang, Phys. Rev. Lett. 66, 1438 (1991).

${ }^{6}$ G. Galli and M. Parrinello, Phys. Rev. Lett. 69, 3547 (1992).

${ }^{7}$ X. P. Li, R. W. Nunes, and D. Vanderbilt, Phys. Rev. B 47, 10891 (1993).

${ }^{8}$ P. Ordejon, D. A. Drabold, R. M. Martin, and M. P. Grumbach, Phys. Rev. B 51, 1456 (1995).

${ }^{9}$ E. Hernandez and M. J. Gillan, Phys. Rev. B 51, 10157 (1995).

${ }^{10}$ J. L. Fattebert and J. Bernholc, Phys. Rev. B 62, 1713 (2000).

${ }^{11}$ C. K. Skylaris, A. A. Mostofi, P. D. Haynes, O. Dieguez, and M. C. Payne, Phys. Rev. B 66, 035119 (2002).

${ }^{12}$ Y. Liu, D. A. Yarne, and M. E. Tuckerman, Phys. Rev. B 68, 125110 (2003).

${ }^{13}$ J. L. Fattebert and F. Gygi, Phys. Rev. B 73, 115124 (2006).

${ }^{14}$ R. Takayama, T. Hoshi, T. Sogabe, S. L. Zhang, and T. Fujiwara, Phys. Rev. B 73, 165108 (2006).

${ }^{15}$ C. K. Skylaris, P. D. Haynes, A. A. Mostofi, and M. C. Payne, J. Chem. Phys. 122, 084119 (2005).

${ }^{16}$ P. D. Haynes, C. K. Skylaris, A. A. Mostofi, and M. C. Payne, Phys. Status Solidi B 243, 2489 (2006).

${ }^{17}$ N. D. M. Hine, P. D. Haynes, A. A. Mostofi, K. Skylaris, and M. C. Payne, Comput. Phys. Commun. 180, 1041 (2009).

${ }^{18}$ D. R. Bowler, R. Choudhury, M. J. Gillan, and T. Miyazaki, Phys. Status Solidi B 243, 989 (2006).

${ }^{19}$ M. J. Gillan, D. R. Bowler, A. S. Torralba, and T. Miyazaki, Comput. Phys. Commun. 177, 14 (2007).

${ }^{20}$ J. M. Soler, E. Artacho, J. D. Gale, A. Garcia, J. Junquera, P. Ordejon, and D. Sanchez-Portal, J. Phys.: Condens. Matter 14, 2745 (2002).

${ }^{21}$ T. Ozaki and H. Kino, Phys. Rev. B 72, 045121 (2005).

${ }^{22}$ L. Genovese, S. Goedecker, A. Neelov, M. Ospici, D. Caliste, S. A. Ghasemi, T. Deutsch, and Q. Hill, [http://inac.cea.fr/L_Sim/BigDFT/].

${ }^{23}$ V. Blum, R. Gehrke, F. Hanke, P. Havu, V. Havu, X. Ren, K. Reuter, and M. Scheffler, Comput. Phys. Commun. 180, 2175 (2009).
${ }^{24}$ T. Miyazaki, D. R. Bowler, R. Choudhury, and M. J. Gillan, J. Chem. Phys. 121, 6186 (2004).

${ }^{25}$ A. S. Torralba, D. R. Bowler, T. Miyazaki, and M. J. Gillan, J. Chem. Theory Comput. 5, 1499 (2009).

${ }^{26}$ P. Ordejón, E. Artacho, and J. M. Soler, Phys. Rev. B 53, R10441 (1996).

${ }^{27}$ P. Pulay, Mol. Phys. 17, 197 (1969).

${ }^{28}$ A. A. Mostofi, P. D. Haynes, C. K. Skylaris, and M. C. Payne, J. Chem. Phys. 119, 8842 (2003).

${ }^{29}$ S. J. Clark, M. D. Segall, C. J. Pickard, P. J. Hasnip, M. J. Probert, K. Refson, and M. C. Payne, Z. Kristallogr. 220, 567 (2005).

${ }^{30}$ W. Kohn, Phys. Rev. Lett. 76, 3168 (1996).

${ }^{31}$ E. Prodan and W. Kohn, Proc. Natl. Acad. Sci. USA 102, 11635 (2005).

${ }^{32}$ W. Kohn, Phys. Rev. 115, 809 (1959).

${ }^{33}$ J. des Cloizeaux, Phys. Rev. 135, A698 (1964).

${ }^{34}$ G. Nencieu, Commun. Math. Phys. 91, 81 (1983).

${ }^{35}$ L. He and D. Vanderbilt, Phys. Rev. Lett. 86, 5341 (2001).

${ }^{36}$ J. des Cloizeaux, Phys. Rev. 135, A685 (1964).

${ }^{37}$ S. Ismail-Beigi and T. A. Arias, Phys. Rev. Lett. 82, 2127 (1999).

${ }^{38}$ R. McWeeny, Rev. Mod. Phys. 32, 335 (1960).

${ }^{39} \mathrm{H}$. Hellman, Einführung in die Quanten Theorie (Deuticke, Leipzig, 1937), p. 285.

${ }^{40}$ R. P. Feynman, Phys. Rev. 56, 340 (1939).

${ }^{41}$ C. K. Skylaris, P. D. Haynes, A. A. Mostofi, and M. C. Payne, Phys. Status Solidi B 243, 973 (2006).

${ }^{42}$ P. D. Haynes, C. K. Skylaris, A. A. Mostofi, and M. C. Payne, Chem. Phys. Lett. 422, 345 (2006).

${ }^{43}$ C. K. Skylaris, A. A. Mostofi, P. D. Haynes, C. J. Pickard, and M. C. Payne, Comput. Phys. Commun. 140, 315 (2001).

${ }^{44}$ A. A. Mostofi, C. K. Skylaris, P. D. Haynes, and M. C. Payne, Comput. Phys. Commun. 147, 788 (2002).

${ }^{45}$ L. Kleinman and D. M. Bylander, Phys. Rev. Lett. 48, 1425 (1982).

${ }^{46}$ U. Essmann, L. Perera, M. L. Berkowitz, T. Darden, H. Lee, and L. G. Pedersen, J. Chem. Phys. 103, 8577 (1995).

${ }^{47}$ J. W. Perram, H. G. Petersen, and S. W. D. Leeuw, Mol. Phys. 65, 875 (1988).

${ }^{48}$ N. Choly and E. Kaxiras, Phys. Rev. B 67, 155101 (2003).

${ }^{49}$ L. Hung and E. A. Carter, Chem. Phys. Lett. 475, 163 (2009).

${ }^{50}$ L. Greengard and V. Rokhlin, J. Comput. Phys. 73, 325 (1987). 
${ }^{51}$ S. G. Louie, S. Froyen, and M. L. Cohen, Phys. Rev. B 26, 1738 (1982).

${ }^{52}$ A. Dal Corso, S. Baroni, R. Resta, and S. de Gironcoli, Phys. Rev. B 47, 3588 (1993).

${ }^{53}$ J. Bernholc, E. L. Briggs, D. J. Sullivan, C. J. Brabec, M. B. Nardelli, K. Rapcewicz, C. Roland, and M. Wensell, Int. J. Quantum Chem. 65, 531 (1997).

${ }^{54}$ E. Artacho, E. Anglada, O. Dieguez, J. D. Gale, A. Garcia, J. Junquera, R. M. Martin, P. Ordejon, J. M. Pruneda, D. SanchezPortal, and J. M. Soler, J. Phys.: Condens. Matter 20, 064208 (2008).

${ }^{55}$ J. L. Fattebert and F. Gygi, Comput. Phys. Commun. 162, 24 (2004).

${ }^{56}$ B. G. Pfrommer, M. Côté, S. G. Louie, and M. L. Cohen, J. Comput. Phys. 131, 233 (1997).

${ }^{57}$ J. Nocedal and S. J. Wright, Numerical Optimization (SpringerVerlag, New York, 2006).

${ }^{58}$ N. Troullier and J. L. Martins, Phys. Rev. B 43, 1993 (1991).

${ }^{59}$ J. P. Perdew and A. Zunger, Phys. Rev. B 23, 5048 (1981).

${ }^{60}$ C. K. Skylaris, P. D. Haynes, A. A. Mostofi, and M. C. Payne, J. Phys.: Condens. Matter 17, 5757 (2005).

${ }^{61}$ C.-K. Skylaris and P. D. Haynes, J. Chem. Phys. 127, 164712 (2007).

${ }^{62}$ A. Ramstad, G. Brocks, and P. J. Kelly, Phys. Rev. B 51, 14504 (1995).
${ }^{63}$ G. J. Kleywegt and T. A. Jones, Acta Crystallogr. Sect. D 54, 1119 (1998).

${ }^{64}$ T. Macke and D. Case, Molecular Modeling of Nucleic Acids (Cambridge University Press, Washington, DC, 1998), Chap. "Modeling unusual nucleic acid structures."

${ }^{65}$ D. Case, T. Darden, T. Cheatham III, C. Simmerling, J. Wang, R. Duke, R. Luo, R. Walker, W. Zhang, K. Merz, B. Roberts, B. Wang, S. Hayik, A. Roitberg, G. Seabra, I. Kolossváry, K. Wong, F. Paesani, J. Vanicek, X. Wu, S. Brozell, T. Steinbrecher, H. Gohlke, Q. Cai, X. Ye, J. Wang, M.-J. Hsieh, G. Cui, D. Roe, D. Mathews, M. Seetin, C. Sagui, V. Babin, T. Luchko, S. Gusarov, A. Kovalenko, and P. Kollman, AMBER 11, University of California, San Francisco, 2010.

${ }^{66}$ N. D. M. Hine, P. D. Haynes, A. A. Mostofi, and M. C. Payne, J. Chem. Phys. 133, 114111 (2010).

${ }^{67}$ S. Goedecker, F. Lançon, and T. Deutsch, Phys. Rev. B 64, 161102(R) (2001).

${ }^{68}$ M. V. Fernández-Serra, E. Artacho, and J. M. Soler, Phys. Rev. B 67, 100101(R) (2003).

${ }^{69}$ K. Nemeth and M. Challacombe, J. Chem. Phys. 121, 2877 (2004).

${ }^{70}$ K. Nemeth and M. Challacombe, J. Chem. Phys. 123, 194112 (2005). 